\title{
Efficacy of airway pressure release ventilation for acute respiratory distress syndrome: a systematic review with meta-analysis
}

\author{
Changqin Chen^, Junhai Zhen^, Shijin Gong^, Jing Yan^, Li Li^ \\ Department of Critical Care Medicine, Zhejiang Hospital, Hangzhou, China \\ Contributions: (I) Conception and design: L Li, J Yan; (II) Administrative support: C Chen, J Zhen; (III) Provision of study materials or patients: S \\ Gong, J Yan, C Chen; (IV) Collection and assembly of data: C Chen, J Zhen, J Yan; (V) Data analysis and interpretation: S Gong, L Li, C Chen, J \\ Yan; (VI) Manuscript writing: All authors; (VII) Final approval of manuscript: All authors. \\ Correspondence to: Dr. Li Li. Department of Critical Care Medicine, Zhejiang Hospital, Hangzhou 310013, China. Email: lilihbch@163.com.
}

\begin{abstract}
Background For many years, airway pressure release ventilation (APRV) has been used to manage patients with lung conditions such as acute respiratory distress syndrome (ARDS). However, it is still unclear whether APRV improves outcomes in critically ill ARDS patients who have been admitted to an intensive care unit (ICU).

Methods: In this study, randomized controlled trials (RCTs) were used to compare the efficacy of APRV to traditional modes of mechanical ventilation. RCTs were sourced from PubMed, Cochrane, and Embase databases (the last dates from August 8, 2019). The Cochrane Handbook for Systematic Reviews of Interventions was used to assess the risk of bias. The relative risk (RR), mean difference (MD), and 95\% confidence intervals (CI) were then determined. Article types such as observational studies, case reports, animal studies, etc., were excluded from our meta-analysis. In total, the data of 6 RCTs and 360 ARDS patients were examined.
\end{abstract}

Results: Six studies with 360 patients were included, our meta-analysis showed that the mean arterial pressure (MAP) in the APRV group was higher than that in the traditional mechanical ventilation group $(\mathrm{MD}=2.35,95 \% \mathrm{CI}: 1.05-3.64, \mathrm{P}=0.0004)$. The peak pressure (Ppeak) was also lower in the APRV group with a statistical difference noted ( $\mathrm{MD}=-2.04,95 \% \mathrm{CI}:-3.33$ to $-0.75, \mathrm{P}=0.002)$. Despite this, no significant beneficial effect on the oxygen index $\left(\mathrm{PaO}_{2} / \mathrm{FiO}_{2}\right)$ was shown between the two groups $(\mathrm{MD}=26.24,95 \% \mathrm{CI}$ : -26.50 to $78.97, \mathrm{P}=0.33$ ). Compared with conventional mechanical ventilation, APRV significantly improved 28-day mortality ( $R R=0.66,95 \%$ CI: $0.47-0.94, \mathrm{P}=0.02$ ).

Discussion: All the included studies were considered to have an unclear risk of bias. We determined that for critically ill patients with ARDS, the application of APRV is associated with an increase in MAP. Inversely, a reduction of the airway Ppeak and 28-day mortality was recorded. There was no sufficient evidence to support the idea that APRV is superior to conventional mechanical ventilation in improving $\mathrm{PaO}_{2} / \mathrm{FiO}_{2}$.

Keywords: Airway pressure release ventilation (APRV); acute respiratory distress syndrome (ARDS); metaanalysis; critically ill patients; randomized controlled trials (RCTs)

Submitted Mar 30, 2021. Accepted for publication Jul 13, 2021.

doi: $10.21037 / \mathrm{apm}-21-747$

View this article at: https://dx.doi.org/10.21037/apm-21-747

$\wedge$ ORCID: Changqin Chen, 0000-0001-5109-114x; Junhai Zhen, 0000-0002-1668-8183; Shijin Gong, 0000-0003-2936-1451; Jing Yan, 0000-0002-6001-7635; Li Li, 0000-0003-3149-6646. 


\section{Introduction}

Acute respiratory distress syndrome (ARDS) is a common cause of acute respiratory failure in patients admitted to an intensive care unit (ICU). ARDS is characterized by pulmonary edema, hyaline membrane formation, atelectasis with progressive respiratory distress, and refractory hypoxemia. Mechanical ventilation has been applied in critically ill patients with ARDS for a long time to promote lung recruitment, improve lung compliance, and increase lung ventilation and gas exchange. Many mechanical ventilation strategies have been used for ARDS patients, such as low tidal volume ventilation (LTVV), positive end-expiratory pressure (PEEP), permissive hypercapnia ventilation (PHV), and high-frequency oscillatory ventilation (HFOV). Despite these strategies, the present mortality rate of ARDS is still as high as $40 \%$ (1). Therefore, we must seek a more effective mode of mechanical ventilation for the management of ARDS.

Airway pressure release ventilation (APRV) has been described for more than 30 years, with Stock et al. (2) first conceiving of APRV in 1987. APRV is a pressure control system that aims to control lung ventilation through different levels of pressure. When used, it is first set at a high airway pressure (Phigh) before being quickly and briefly adjusted to a lower level (usually $0 \mathrm{cmH}_{2} \mathrm{O}$, Plow). This switching between Phigh and Plow allows for the partial contraction of the lungs to prevent unstable alveolar collapse. APRV can also prevent further collapse of unstable alveolar units and cause further alveolar recruitment in damaged lung tissue. Reports have shown that APRV results in airways having a lower peak pressure (Ppeak) and mean pressure than traditional mechanical ventilation modes (3).

Furthermore, it has other physiological benefits, such as improving clinical outcomes for hemodynamic changes and respiratory dynamics. Recently, Han et al. (4) found that APRV effectively improved oxygenation and kept hemodynamic stability compared with low tidal volume (LTV) in canines with ARDS. In clinical practice, there has also been evidence that APRV improves a patient's oxygenation index $\left(\mathrm{PaO}_{2} / \mathrm{FiO}_{2}\right)$ and reduces the impact of ARDS on circulation and mortality when compared with other modes of mechanical ventilation $(5,6)$. However, Maxwell et al. (7) found that there is no statistical evidence to support this claim, with APRV and other ventilation modes resulting in similar patient outcomes for critically ill ARDS adults, e.g., ventilator days, length of stay, ventilatorassociated pneumonia, and mortality. Therefore, there is still controversy around whether APRV is more effective than traditional mechanical ventilation modes. More recently, several randomized controlled trials (RCTs) were published investigating the effect of APRV on critically ill patients with ARDS. In this study, we systematically analyze the results provided by these trials. We present the following article in accordance with the PRISMA reporting checklist (available at https://dx.doi.org/10.21037/apm-21-747).

\section{Methods}

\section{Literature search strategy}

According to an inclusion/exclusion criteria, two researchers independently assessed relevant literature published in PubMed, Cochrane, and Embase databases. This included any material published between the establishment of the databases and August 8, 2019. The study was not restricted to any language. The search was performed using the following items: airway pressure release ventilation/APRV, acute respiratory distress syndrome/acute lung injury/ ARDS/shock lung, randomized controlled trials/RCT. Disagreements between the researchers were resolved through discussions, and when discussions failed to reconcile the disagreements, a third researcher was included to assist in making the final decision.

\section{Inclusion criteria}

\section{Research type}

RCTs were used to compare the efficacy of APRV to traditional modes of mechanical ventilation.

\section{Study participants}

Critically ill patients aged 18 years and older admitted to ICU with ARDS. This met the Berlin criteria for ARDS published by the European Resuscitation Council (ERC), the European Society of Intensive Care Medicine (ESICM), and the American Thoracic Society (ATS) in 2012.

\section{Intervention measures}

Patients in the APRV group received APRV, while patients in the control intervention group received traditional mechanical ventilation modes, including synchronized intermittent mandatory ventilation (SIMV), LTVV, controlled mechanical ventilation (CMV), and assistantcontrol (AC) ventilation. The mechanical ventilation modes used in the two groups did not overlap, and all were parallel 
control trials.

\section{Outcome measures}

Twenty-eight-day mortality, oxygenation index $\left(\mathrm{PaO}_{2} /\right.$ $\left.\mathrm{FiO}_{2}\right)$, millimeters of mercury $(\mathrm{mmHg})$, mean arterial pressure (MAP), airway peak pressure (Ppeak).

\section{Exclusion criteria}

Observational studies, quasi-randomized or crossover studies, case reports, animal studies, pediatric population studies, review articles, and repeated reports were all excluded from our analysis.

\section{Data extraction}

Two researchers independently obtained data according to the inclusion and exclusion criteria. From this, we created our data extraction tables to categorize the relevant data. These tables included the following information: (I) basic publication information: author names, time and place of publication; (II) study participants: sample size, sex, age; (III) intervention measures: which mechanical ventilation modes were used; (IV) research results: 28-day mortality, $\mathrm{PaO}_{2} /$ $\mathrm{FiO}_{2}, \mathrm{MAP}$, airway Ppeak.

\section{Methodological quality assessment}

The assessment of the risk of bias was conducted following The Cochrane Handbook for Systematic Reviews of Interventions. This involved considering various aspects, including random sequence generation, allocation concealment, blinding of patients and personnel, blinding of outcome assessment, incomplete outcome data, selective reporting, and other sources of bias. From this, we established assessment criteria that defined whether high risk, low risk, or unclear risk of bias was present.

\section{Statistical analysis}

Studies were collated and checked to ensure they met the requirements of meta-analysis. Data analysis was then performed by using Cochrane Review Manager software (RevMan 5.3, Cochrane, London, UK). Using a two-sided test, the significance level was 0.05 , with $\mathrm{P}<0.05$ considered statistically significant. Metrological data (Ppeak, MAP, $\mathrm{PaO}_{2} / \mathrm{FiO}_{2}$ ) was analyzed through mean deviation (MD) and standard deviation (SD) while counting data (28-day mortality) was analyzed with the relative risk (RR) and $95 \%$ confidence intervals $(95 \% \mathrm{CI})$ as an effective statistic. If a study was found to have no significant heterogeneity $\left(\mathrm{I}^{2}<50 \%, \mathrm{P}>0.05\right)$, we used the Peto Mantel-Haenszel fixedeffects model. If significant $\left(\mathrm{I}^{2} \geq 50 \%, \mathrm{P}<0.05\right)$, we used the DerSimonian-Laird (DSL) random-effects model. Reverse funnel plots were used to assess potential publication bias. Where necessary, we also performed a sensitivity analysis to assess the review results.

\section{Results}

\section{Literature retrieval}

We searched through a total of 367 articles: 224 in PubMed, 120 in Embase, 16 in Cochrane, and 7 in other sources (such as abstracts from conferences). After removing duplicates, we were left with 197 articles. After reading through abstracts, further articles were excluded due to observational studies, case reports, animal studies, pediatric population studies, review articles, and repeated report reviews. Following this, 8 articles remained, and the full text of each was read in detail. Two studies were removed for lacking relevant outcome indications or for displaying too short of an observation time. This left us with the 6 studies used in our analysis (Figure 1).

\section{Basic information of the RCTs included}

The key baseline characteristics of the RCTs are illustrated in Table 1. The publication time of these studies ranged from January 2003 to December 2017. All trials were parallel control trials. The nationalities of the studies included Asian, European, and American. The outcome indications included 28-day mortality, $\mathrm{PaO}_{2} / \mathrm{FiO}_{2}, \mathrm{MAP}$, and airway Ppeak. A total of 360 patients were included in the study, including 172 patients in the APRV group and 188 patients in the mechanical ventilation control group. The sample size ranged from 22-138. The age of patients was between $30-80$ years. Details of the baseline characteristics of study participants are shown in Table 2 . As for the initial setting of the mechanical ventilation, most patients in the control group received SIMV, and the strategy of lung-protective ventilation was also adopted. While in the APRV group, parameter settings were slightly varied in different trials for there is still no consensus on how the APRV should be set. Most studies set Phigh $\leq 30 \mathrm{cmH}_{2} \mathrm{O}$ or UIP (upper inflection point in pressure- 


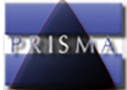

PRISMA 2009 Flow Diagram
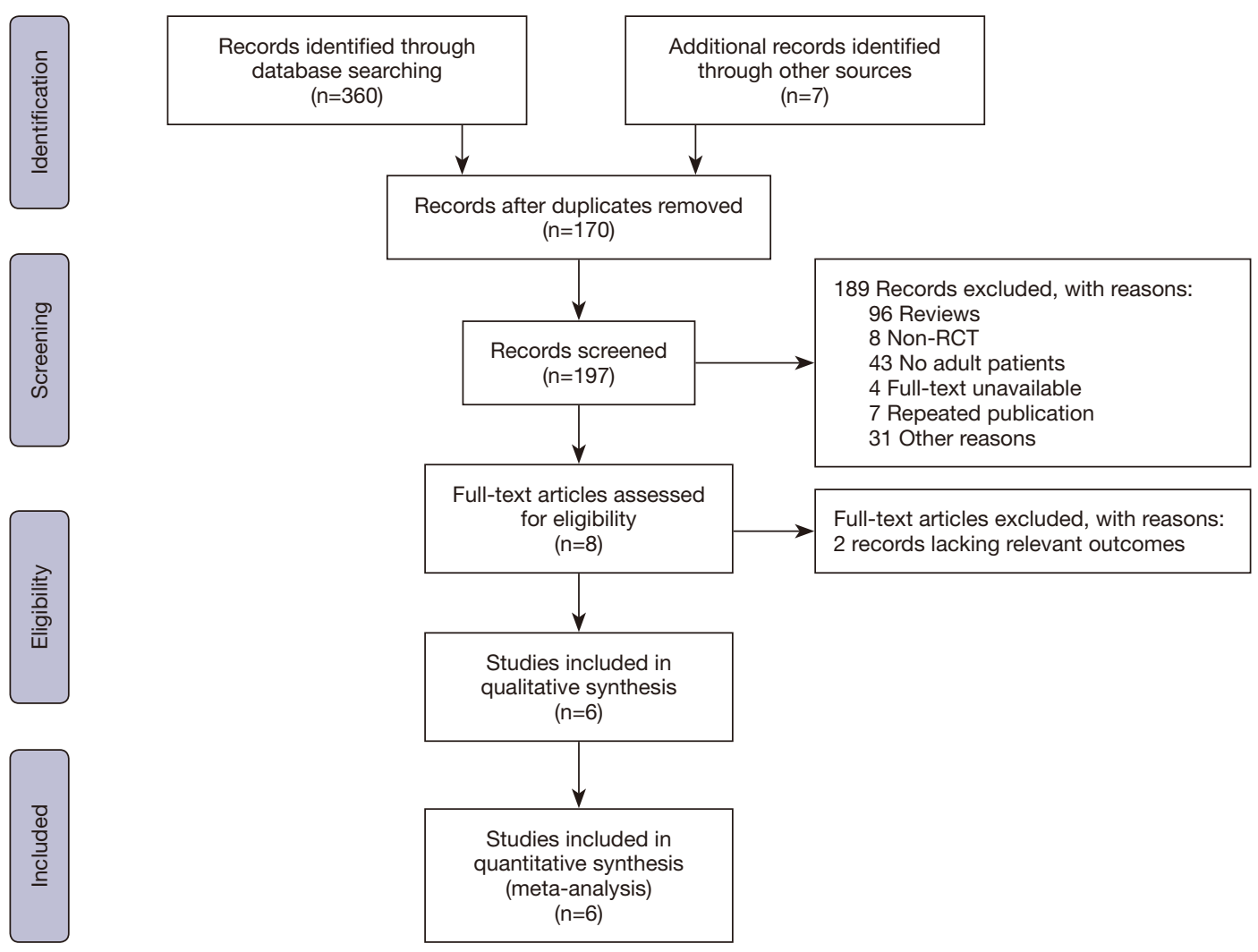

Figure 1 PRISMA flow diagram of the study selection process.

Table 1 The basic information of the studies

\begin{tabular}{|c|c|c|c|c|c|c|c|}
\hline Studies included & $\begin{array}{l}\text { Intervention } \\
\text { measures }\end{array}$ & $\begin{array}{l}\text { Sample } \\
\text { size }\end{array}$ & $\begin{array}{c}\text { Male and } \\
\text { female ratios }\end{array}$ & \multicolumn{2}{|c|}{ Age } & Country & Outcomes \\
\hline Zhou Y 2017 & APRV + LTV & 138 & $91 / 47$ & $51.5 \pm 15.0$ & $52.0 \pm 15.1$ & China & $\begin{array}{l}\mathrm{PaO}_{2} / \mathrm{FiO}_{2}, 28 \text {-day mortality, } \\
\text { MAP, Ppeak }\end{array}$ \\
\hline Li JQ 2016 & APRV + SIMV & 52 & NA & $54.3 \pm 8.4$ & $53.6 \pm 9.5$ & China & $\begin{array}{l}\mathrm{PaO}_{2} / \mathrm{FiO}_{2}, 28 \text {-day mortality, } \\
\text { MAP, Ppeak }\end{array}$ \\
\hline Varpula T 2003 & APRV + SIMV-PC/PS & 33 & $25 / 8$ & $50.0(37.0-60.0)$ & $46.5(37.2-55.3)$ & Finland & 28-day mortality \\
\hline Ota K 2009 & APRV + SIMV & 57 & NA & NA & NA & USA & $\mathrm{PaO}_{2} / \mathrm{FiO}_{2}$, 28-day mortality \\
\hline Song S 2016 & APRV + SIMV & 22 & $13 / 9$ & $63[40,73]$ & $73[53,80]$ & China & $\mathrm{PaO}_{2} / \mathrm{FiO}_{2}, \mathrm{MAP}$, Ppeak \\
\hline
\end{tabular}

APRV, airway pressure release ventilation; LTV, low tidal volume; SIMV, synchronized intermittent mandatory ventilation; PC, pressure control; PS, pressure support; NA, not available; MAP, mean artery pressure; Ppeak, peak of pressure. 
Table 2 The detailed information of the studies

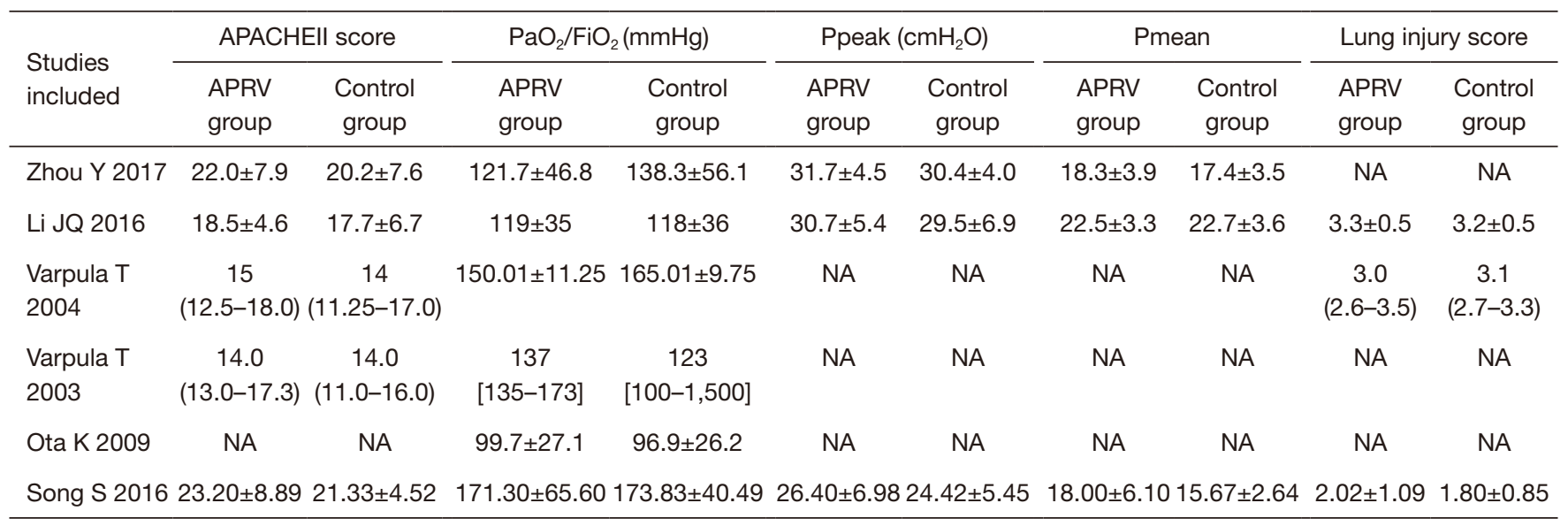

APRV, airway pressure release ventilation; NA, not available; MAP, mean artery pressure; Pmean, mean pressure; Ppeak, peak pressure.

volume curve) in order to limit excessive airway pressure, in some studies $(1,5,8)$, the Thigh was set at about $4(2.5-8)$ seconds, and the other studies $(6,9,10)$ set the Thigh according to the frequency of pressure shifts (the release frequency was $10-14$ cycles/min in most trails). As for Plow, 2 studies $(1,8)$ used $0 \mathrm{cmH}_{2} \mathrm{O}$, another 2 studies conducted by Varpula et al. $(6,10)$ adopted PEEP set before as the level of Plow; however, 2 RCTs conducted in China used $5 \mathrm{cmH}_{2} \mathrm{O}(9)$ and LIP (lower inflection point in the pressurevolume curve) (5) as the value of Plow respectively. In Zhou's (9) study, they set Tlow adjusted to terminate the peak expiratory flow rate to $\geq 50 \%, 2$ studies set Tlow to $0.4-0.8$ seconds (1) and 1 second (5) respectively, in Ota's study (8), Tlow was the difference of the time for one respiratory cycle and Thigh, the last 2 studies set the Tlow according to the frequency of pressure shifts. The detailed initial setting of the mechanical ventilation in both groups is shown in Table 3.

\section{Results of methodological quality evaluation}

After the methodological quality assessment of the 6 included studies, none were judged to have a low or high risk of bias, resulting in each having an unclear risk of bias (Figure 2).

\section{Results of the meta-analysis}

\section{MAP}

Four RCTs were included in the MAP analysis $(1,5,9,10)$. This comprised 137 patients in the APRV group and 162 in the control group. No statistical heterogeneity was found between the two groups $\left(\mathrm{P}=0.41 ; \mathrm{I}^{2}=0\right)$. Using the Peto Mantel-Haenszel fixed-effects model, the result showed there was a statistical difference for MAP (MD $=2.35,95 \%$ CI: $1.05-3.64, \mathrm{P}=0.0004)$. Figure 3 exemplifies that the APRV group had a higher MAP than that of the control group.

\section{$\mathrm{PaO}_{2} / \mathrm{FiO}_{2}$}

For the analysis of $\mathrm{PaO}_{2} / \mathrm{FiO}_{2}, 5$ RCTs were included $(1,5,8-10)$ : 154 patients in the APRV group, 173 in the control group. Statistical heterogeneity was found between these two groups $\left(\mathrm{P}<0.00001, \mathrm{I}^{2}=93 \%\right)$, and so the DSL random-effects model was used to analyze the results. This showed no statistical difference for $\mathrm{PaO}_{2} / \mathrm{FiO}_{2}$ between the two groups ( $M D=26.24,95 \% \mathrm{CI}:-26.50$ to $78.97, \mathrm{P}=0.33$ ) (Figure 4).

\section{Airway Ppeak}

There were only 2 studies included in this analysis $(1,9)$, with 97 patients in the APRV group and 93 in the control group. Because statistical heterogeneity was shown between the two groups $\left(\mathrm{P}=0.39, \mathrm{I}^{2}=0\right)$, we used the Peto Mantel-Haenszel fixed-effects model. The result showed there was a statistical difference between the two groups for airway Ppeak $(\mathrm{MD}=-2.04,95 \% \mathrm{CI}:-3.33$ to $-0.75, \mathrm{P}=0.002$ ) (Figure 5).

\section{8-day mortality}

In regards to 28 -day mortality, 5 RCTs were included $(1,6,8-10)$. There were 162 patients in the APRV group and 176 patients in the control group. We again used the Peto Mantel-Haenszel fixed-effects model because of no 
Table 3 The initial setting of the mechanical ventilation in both groups

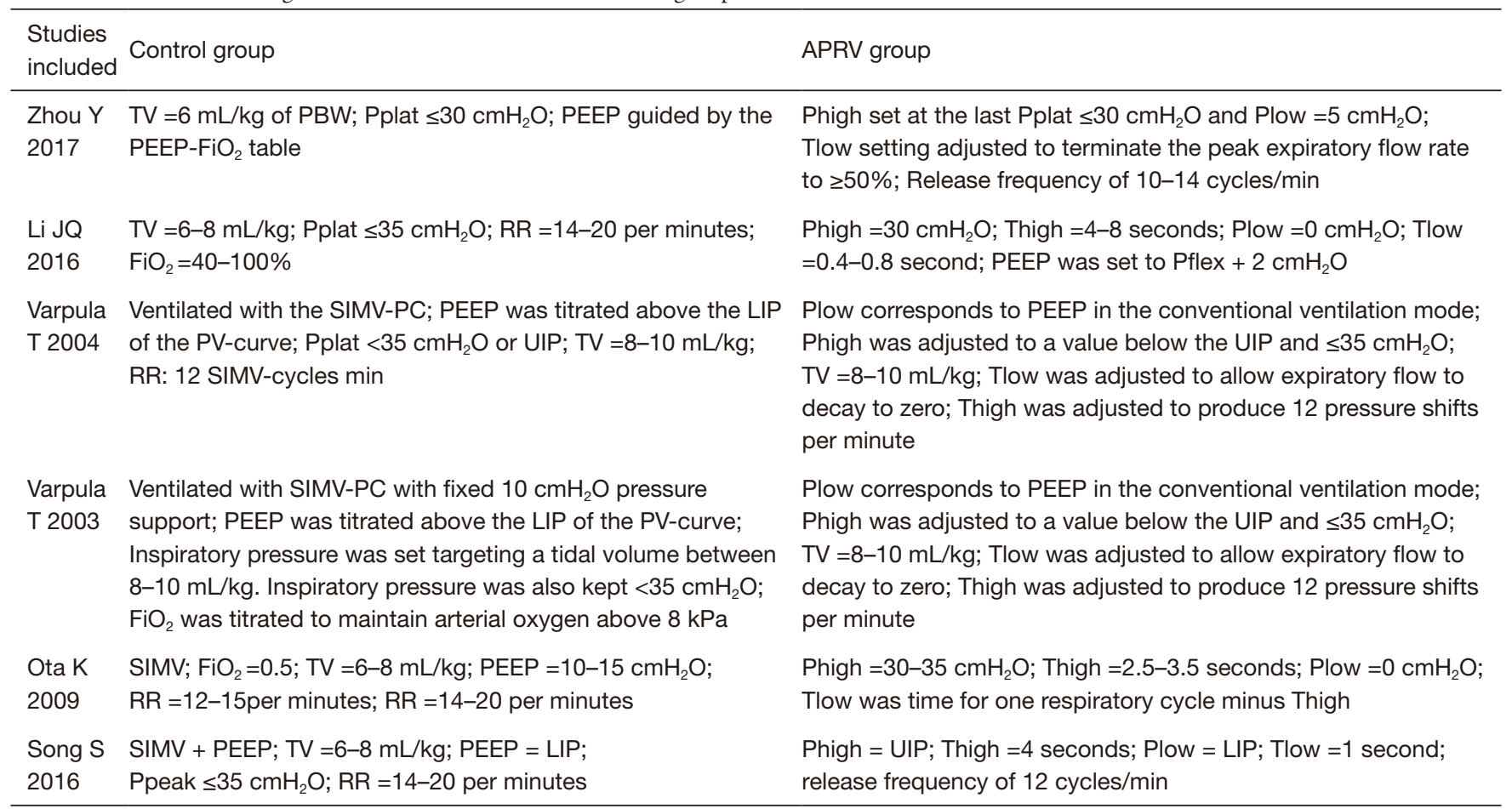

TV, tidal volume; PBW, predicted body weight; Phigh, high airway pressure; Thigh, time of high duration; Plow, low airway pressure; Tlow, time of low duration; Pplat, plateau airway pressure; RR, respiratory rate; Pflex, lower inflection point of the quasi-static PV curve; LIP, lower inflection point; UIP, upper inflection point.

statistical heterogeneity $\left(\mathrm{P}=0.82, \mathrm{I}^{2}=0\right)$. Subsequently, we found there was a significant statistical difference between the two groups for 28 -day mortality $(\mathrm{RR}=0.66,95 \% \mathrm{CI}$ : $0.47-0.94, \mathrm{P}=0.02$ ) (Figure 6). Through this, we determined that the mortality rate of ARDS patients with APRV was $34 \%$ lower than that of the control group.

\section{Sensitivity and publication bias analysis}

Figure 4 exemplifies the heterogeneity between the two groups $\left(\mathrm{P}<0.00001, \mathrm{I}^{2}=93 \%\right)$, but to find the potential sources of this heterogeneity, we used leave-one-out sensitivity analysis. However, the removal of any study failed to reduce the value of $\mathrm{I}^{2}$ below $50 \%$. As a result, we found that the heterogeneity between the two groups did not derive from any one study. In Table 1, a significant difference can be seen in each study's sample size, from 22 to 138. Moreover, the baseline of $\mathrm{PaO}_{2} / \mathrm{FiO}_{2}$ varied among different studies, especially in that of Ota et al. (8), which may indicate one of the potential causes of heterogeneity. We also assessed the publication bias for $\mathrm{PaO}_{2} / \mathrm{FiO}_{2}$ through a funnel plot, using the RR value as the horizontal abscissa and the standard error (SE) value as the ordinate. The subsequent funnel plot was not visually symmetrical, suggesting the possibility of publication bias (Figure 7).

\section{Discussion}

Our systematic review concentrated on determining the effect of APRV on critically ill patients with ARDS. In total, 6 RCTs were used, with a total of 360 patients included in the meta-analysis. The major findings of our review suggest that the application of APRV reduces airway Ppeak, has less impact on the circulation of critically ill ARDS patients (APRV group had a higher MAP), and results in lower 28-day mortality.

For traditional mechanical ventilation modes, one can set a respiratory rate, pressure level, tidal volume, positive endexpiratory pressure (PEEP), and other parameters. APRV differs from traditional mechanical ventilation modes as it provides only pressure-limited and time-cycled biphasic positive airway pressure (BIPAP) ventilation. This means 


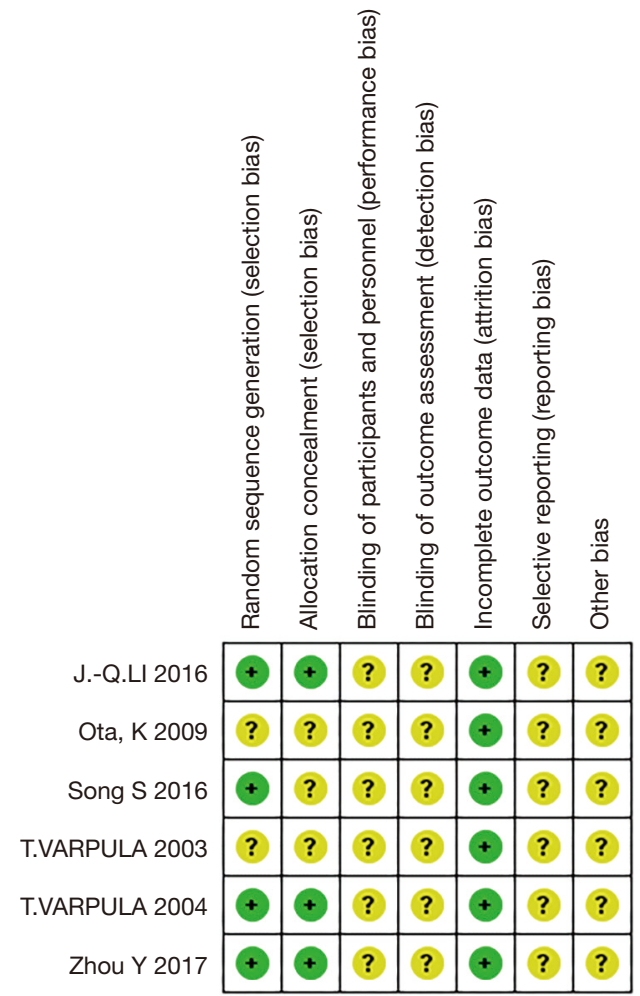

Figure 2 Results of methodological quality evaluation. All studies belonged to the unclear risk of bias. that its parameter settings allow for high airway pressure levels (Phigh; $20-35 \mathrm{cmH}_{2} \mathrm{O}$, generally less than $35 \mathrm{cmH}_{2} \mathrm{O}$ ) and duration (Thigh; 4-6 seconds), and low airway pressure levels (Plow; generally 0) and duration (Tlow; Defined as the time when the expiratory flow rate decreases to $75 \%$ of the peak expiratory flow rate) (11). Internationally, there is still a lack of consistency in how APRV is applied.

From a theoretical perspective, APRV is useful in promoting lung recruitment and maintaining an open alveolar due to the Thigh being longer than the Tlow. In animal models, it has also been shown that compared with other ventilation modes, APRV can effectively preserve the concentration of surfactant proteins A and B in APRV groups at the early stage of ARDS and acute lung injury (ALI) $(12,13)$. Yoshida et al. (14) verified APRV was more efficient than pressure support ventilation (PSV) in decreasing atelectasis in ARDS patients through the analysis of 3-dimensional reconstruction and volumetric computed tomography (CT) data. However, whether APRV is truly more effective in improving $\mathrm{PaO}_{2} / \mathrm{FiO}_{2}$ than other modes is still yet to be determined. This is because results vary between different studies, with some researchers finding no significant difference in $\mathrm{PaO}_{2} / \mathrm{FiO}_{2}$ between APRV and other modes $(1,5,10)$. In 2017 , the largest study to date of the efficacy of APRV in ARDS patients concluded that

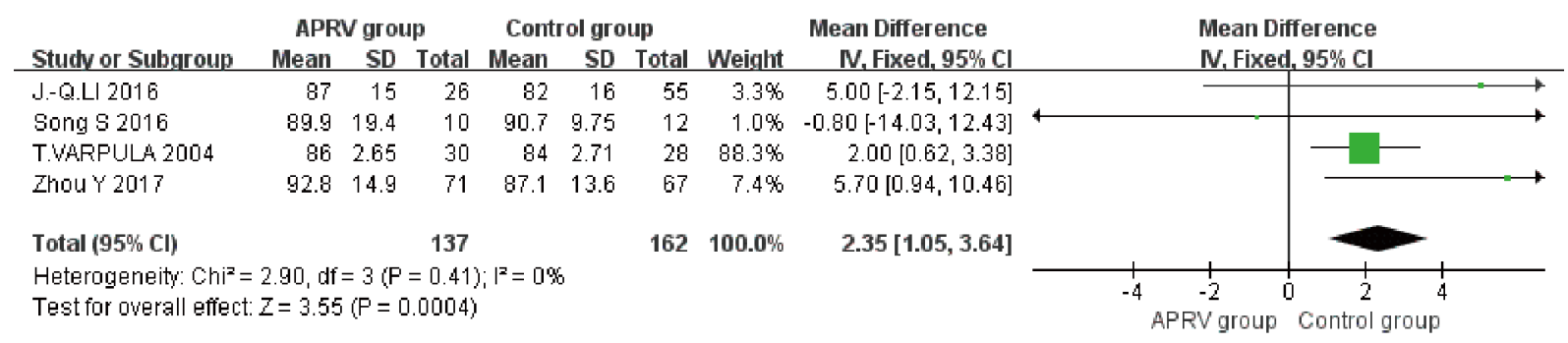

Figure 3 Forest plot comparing MAP among APRV group to that of control group. MAP, mean arterial pressure; APRV, airway pressure release ventilation.

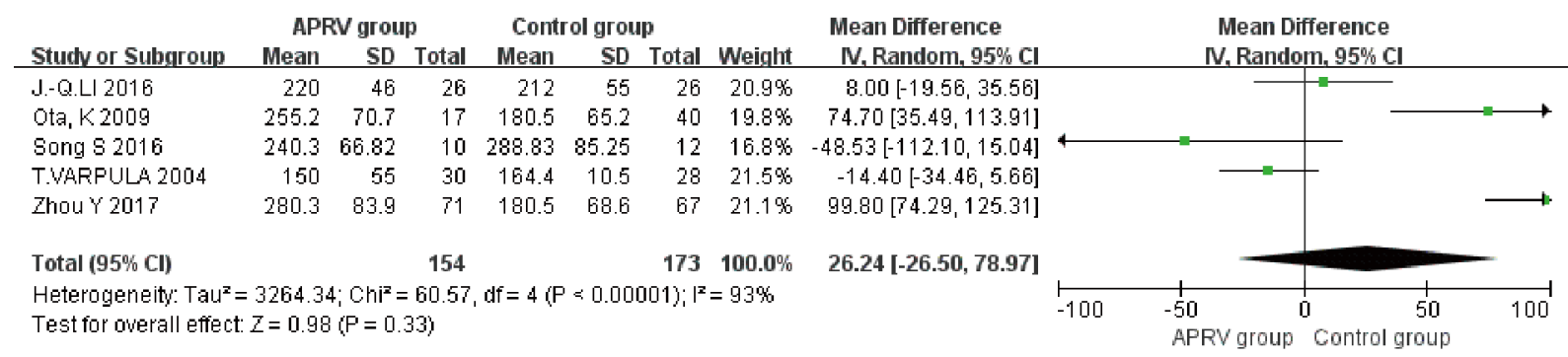

Figure 4 Forest plot comparing $\mathrm{PaO}_{2} / \mathrm{FiO}_{2}$ among APRV group to that of control group. APRV, airway pressure release ventilation. 


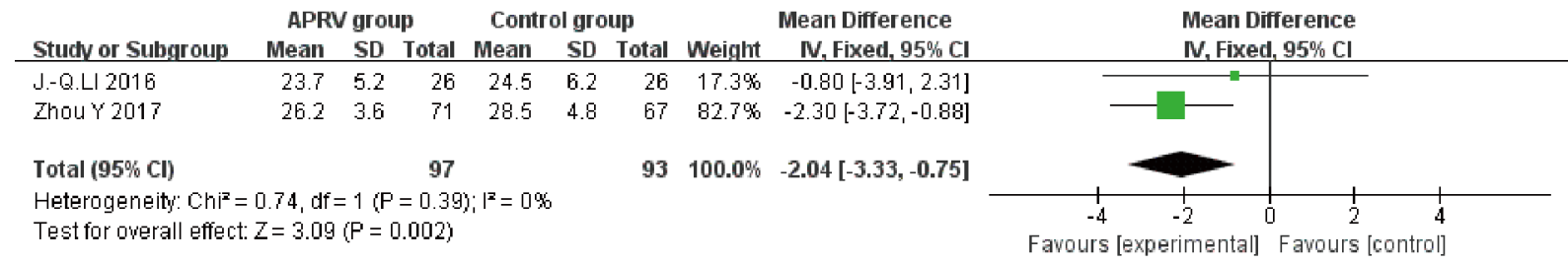

Figure 5 Forest plot comparing the Ppeak among APRV group to that of control group. APRV, airway pressure release ventilation.

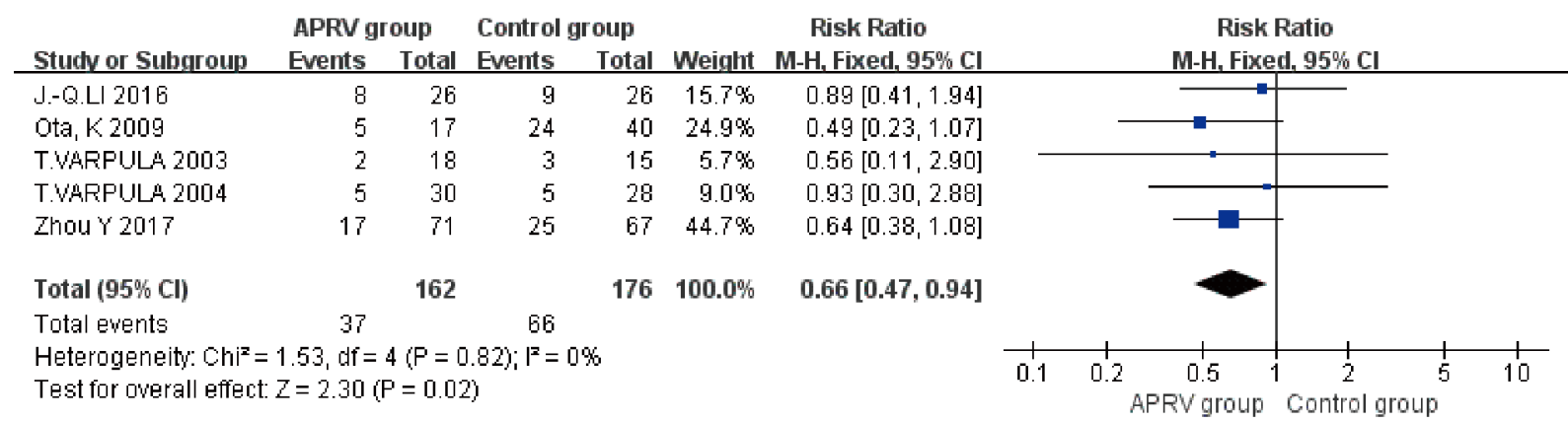

Figure 6 Forest plot comparing 28-day mortality among APRV group to that of control group. APRV, airway pressure release ventilation.

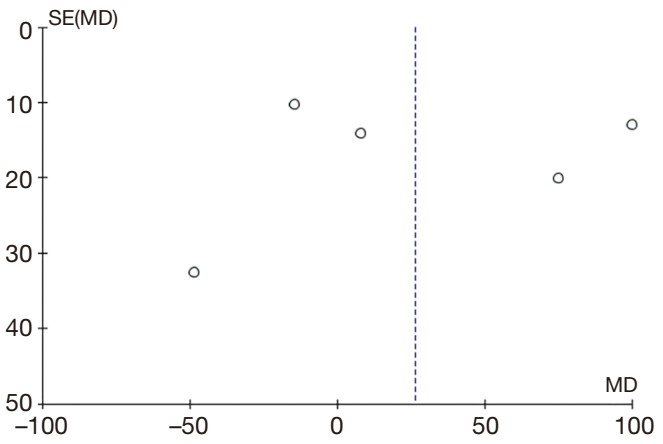

Figure 7 The funnel plot of the publication bias analysis. This funnel plot is not visually symmetrical and reveals potential publication bias. SE, standard error.

when compared with LTVV, APRV did indeed improve $\mathrm{PaO}_{2} / \mathrm{FiO}_{2}$ and respiratory system compliance (9). Our meta-analysis discovered that $\mathrm{PaO}_{2} / \mathrm{FiO}_{2}$ was not higher in the APRV group, although the heterogeneity between the two groups may have influenced the specific result.

Furthermore, there are currently no multicenter RCTs files and only a small number of RCTs available to help assess the effect of APRV in patients' oxygenation. We can see that it is necessary to conduct higher quality and larger sample size studies to further clarify the real effect of APRV in oxygenation among critically ill ARDS patients. It is also important to note that though our study did not show that APRV had any $\mathrm{PaO}_{2} / \mathrm{FiO}_{2}$ advantage over traditional mechanical ventilation modes, lower 28-day mortality in the APRV group was recorded. We concluded that this could stem from many reasons. Firstly, our study did not show that the APRV group recorded a worse $\mathrm{PaO}_{2} / \mathrm{FiO}_{2}$ than other modes. Secondly, our meta-analysis demonstrated a better circulatory status (higher MAP) in the APRV group. And lastly, APRV may have other strengths like ameliorating inflammatory conditions (see below) to improve 28-day mortality.

In addition to assessing the effect of APRV on the respiratory system, we also considered the influence of APRV on the circulatory system. Our meta-analysis showed that a lower airway Ppeak in the APRV group reduced the effect of ARDS on circulation and decreased the incidence of barometric pulmonary injury (15). Kaplan et al. (3) also found that a lower Ppeak with APRV led to a decrease in transmitted intrathoracic pressure, which enhanced venous return and increased the cardiac index (CI). In turn, this leads to a decreased pressor requirement to support MAP and oxygen delivery when compared with pressurecontrolled ventilation (PCV). Other experiments have also shown that APRV can increase the blood flow in organs 
such as the liver (16) and kidney (17), thus improving the perfusion of those organs. In our study, the APRV group had a higher MAP, which further indicates that it has fewer side effects on the circulatory function when compared with other traditional ventilation modes.

APRV also has been shown to have other advantages when applied to ARDS. For example, Habashi's study confirmed that during APRV, ARDS patients maintain their breath, which reduces the requirements of sedation and neuromuscular blockers (18). The result of such spontaneous breathing increases the blood flow of respiratory muscles and prevents the occurrence of respiratory muscle atrophy (19). Different from other mechanical ventilation modes, APRV changes airway pressure to a lower level (Plow) for a short time regularly. During this release stage, it effectively eliminates carbon dioxide (11). Surprisingly, some researchers have even found that APRV may inhibit the production of inflammatory factors, the apoptosis of endothelial cells, the destruction of the extracellular matrix (ECM) mediated by matrix metalloproteinase (MMP), and reduce mortality in animals (20).

Bajaj et al. (21) had conducted a Meta-analysis assessed the efficacy of APRV in critically ill patients compared with traditional ventilation modes in 2015 , there was no significant difference in 28-day mortality of two groups $(\mathrm{OR}=0.74,95 \% \mathrm{CI}=0.38-1.47, \mathrm{P}=0.40)$, Subgroup analysis performed on ALI/ARDS patients (3 studies) also revealed no significant difference in mortality at 28 days $(\mathrm{OR}=0.72$, $95 \% \mathrm{CI}=0.32-1.60, \mathrm{P}=0.42$ ), and the total number of patients was only 249 , the insufficient number of studies included in this meta-analysis may influence the reliability of their result. In 2020, a meta-analysis was conducted by Sun et al. (22), 14 studies with 2096 ARDS patients were included in this meta-analysis; however, most studies included were retrospective studies, they found ARRV was effective in increasing the oxygenation of ARDS patients; however, there were no differences in mortality and duration of ICU stay between APRV and other mechanical ventilation modes. Another meta-analysis published in 2019 found APRV was associated with more ventilatorfree days, lower mortality and no higher proportion of side effects caused by mechanical ventilation were found in the APRV group; however, this meta-analysis included all acute hypoxemic respiratory failure patients, and no further subgroup analysis was conducted to determine the clinical effect of APRV on ARDS patients (23). In Zhong's (24) meta-analysis, they found APRV could reduce mortality, ICU stays, and duration of ventilation and improve the compliance of lung, MAP, and $\mathrm{PaO}_{2} / \mathrm{FiO}_{2}$ compared with other mechanical ventilation modes in ARDS patients. In the systematic review of Andrews et al. (25), Early application of APRV in traumatic patients represented lower mean ARDS incidence (14.0\% vs. $1.3 \%$ ) and in-hospital mortality (14.1\% vs. 3.9\%) compared with traditional mechanical ventilation modes, Unfortunately, all the studies involved were retrospective or observational. In the review of Jain et al. (26), they summarized the APRV in past 30 years, and thought APRV stabilizes alveoli and reduces the incidence of ARDS in clinically relevant animal models and in trauma patients, they regarded APRV as a highly lungprotective ventilation strategy. Although our meta-analysis also found the APRV could increase MAP, reduce the airway Ppeak and 28-day mortality, a strength of our analysis is that we included the newly published RCTs, especially those published in recent years, and 2 studies were included in our meta-analysis that former meta-analysis never included, of which, Song S's RCT showed the $\mathrm{PaO}_{2} / \mathrm{FiO}_{2}$ in APRV group was lower than the control group with a statistical difference $(240.30 \pm 66.82,288.83 \pm 85.25$ respectively, $\mathrm{P}<0.05)$, this may explain why there was no beneficial effect on $\mathrm{PaO}_{2} / \mathrm{FiO}_{2}$ in APRV group in our meta-analysis.

From our study, we found the key strengths of our metaanalysis included: (I) having two researchers independently screen 3 databases and carefully evaluate the quality of each RCTs; (II) using the Cochrane risk of bias tool to assess the methodological quality; (III) including a total of 6 studies, most of which were recently published, and 2 of which were never before included in a meta-analysis; (IV) being the first meta-analysis to evaluate the efficiency of APRV on the airway Ppeak among ARDS patients by assessing ventilator-associated lung injury (the main factor affecting the circulatory system); (V) our study strictly conformed to the broad EQUATOR guidelines--a tool that ensures the value and reliability of the research literature (27). All the strengths mentioned above add to the robustness and clinical value of our meta-analysis.

It is also important to mention the shortcomings of this study, which included: (I) most of the research samples were small; (II) most trials were not blinded and therefore may be biased; (III) in the meta-analysis of $\mathrm{PaO}_{2} / \mathrm{FiO}_{2}$, the heterogeneity among the studies indicates that it has a high publication bias. Such shortcomings may have impacted our outcomes. Therefore, we still need to be cautious with the results of this study, and further research needs to consider the actual situation of the patients involved more closely. 


\section{Conclusions}

To summarize, this study conducted a meta-analysis of 6 RCTs to compare the efficacy of using APRV to traditional mechanical ventilation modes for critically ill ARDS patients. Our study was strengthened by the inclusion of 3 recently published RCTs. Based on our systematic review, our research indicates that when compared to traditional modes, APRV is more effective in reducing airway Ppeak, has less impact on circulation (higher MAP), and improves 28-day mortality. It also leads us to determine that there is no therapeutic benefit in $\mathrm{PaO}_{2} / \mathrm{FiO}_{2}$. Though the number of included studies is still insufficient, we believe higherquality RCTs should be conducted. This would help further clarify the real clinical effect of APRV in ARDS treatment in the future.

\section{Acknowledgments}

Funding: This work was supported by Zhejiang Provincial Program for the Zhejiang Medical and Health Science and Technology Program (2021450592).

\section{Footnote}

Reporting Checklist: The authors have completed the PRISMA reporting checklist. Available at https://dx.doi. org/10.21037/apm-21-747

Conflicts of Interest: All authors have completed the ICMJE uniform disclosure form (available at https://dx.doi. org/10.21037/apm-21-747). The authors have no conflicts of interest to declare.

Ethical Statement: The authors are accountable for all aspects of the work in ensuring that questions related to the accuracy or integrity of any part of the work are appropriately investigated and resolved.

Open Access Statement: This is an Open Access article distributed in accordance with the Creative Commons Attribution-NonCommercial-NoDerivs 4.0 International License (CC BY-NC-ND 4.0), which permits the noncommercial replication and distribution of the article with the strict proviso that no changes or edits are made and the original work is properly cited (including links to both the formal publication through the relevant DOI and the license). See: https://creativecommons.org/licenses/by-nc-nd/4.0/.

\section{References}

1. Li JQ, Li N, Han GJ, et al. Clinical research about airway pressure release ventilation for moderate to severe acute respiratory distress syndrome. Eur Rev Med Pharmacol Sci 2016;20:2634-41.

2. Stock MC, Downs JB, Frolicher DA. Airway pressure release ventilation. Crit Care Med 1987;15:462-6.

3. Kaplan LJ, Bailey H, Formosa V. Airway pressure release ventilation increases cardiac performance in patients with acute lung injury/adult respiratory distress syndrome. Crit Care 2001;5:221-6.

4. Han GJ, Li JQ, Pan CG, et al. Experimental study of airway pressure release ventilation in the treatment of acute respiratory distress syndrome. Exp Ther Med 2017;14:1941-6.

5. Song S, Tian H, Yang X, et al. The clinical effect of airway pressure release ventilation for acute lung injury/acute respiratory distress syndrome. Zhonghua Wei Zhong Bing Ji Jiu Yi Xue 2016;28:15-21.

6. Varpula T, Jousela I, Niemi R, et al. Combined effects of prone positioning and airway pressure release ventilation on gas exchange in patients with acute lung injury. Acta Anaesthesiol Scand 2003;47:516-24.

7. Maxwell RA, Green JM, Waldrop J, et al. A randomized prospective trial of airway pressure release ventilation and low tidal volume ventilation in adult trauma patients with acute respiratory failure. J Trauma 2010;69:501-10; discussion 511.

8. Ota K, Tanigawa K, Otani T, et al. Does airway pressure release ventilation offer benefits over conventional ventilatory supports for severe acute respiratory distress syndrome? Critical Care Medicine 2009;37:A190.

9. Zhou Y, Jin X, Lv Y, et al. Early application of airway pressure release ventilation may reduce the duration of mechanical ventilation in acute respiratory distress syndrome. Intensive Care Med 2017;43:1648-59.

10. Varpula T, Valta P, Niemi R, et al. Airway pressure release ventilation as a primary ventilatory mode in acute respiratory distress syndrome. Acta Anaesthesiol Scand 2004;48:722-31.

11. Andrews P, Habashi N. Airway pressure release ventilation. Curr Probl Surg 2013;50:462-70.

12. Emr B, Gatto LA, Roy S, et al. Airway pressure release ventilation prevents ventilator-induced lung injury in normal lungs. JAMA Surg 2013;148:1005-12.

13. Roy S, Sadowitz B, Andrews P, et al. Early stabilizing alveolar ventilation prevents acute respiratory distress 
syndrome: a novel timing-based ventilatory intervention to avert lung injury. J Trauma Acute Care Surg 2012;73:391-400.

14. Yoshida T, Rinka H, Kaji A, et al. The impact of spontaneous ventilation on distribution of lung aeration in patients with acute respiratory distress syndrome: airway pressure release ventilation versus pressure support ventilation. Anesth Analg 2009;109:1892-900.

15. Hirani A, Marik PE, Plante LA. Airway pressure-release ventilation in pregnant patients with acute respiratory distress syndrome: a novel strategy. Respir Care 2009;54:1405-8.

16. Hering R, Bolten JC, Kreyer S, et al. Spontaneous breathing during airway pressure release ventilation in experimental lung injury: effects on hepatic blood flow. Intensive Care Med 2008;34:523-7.

17. Kamath SS, Super DM, Mhanna MJ. Effects of airway pressure release ventilation on blood pressure and urine output in children. Pediatr Pulmonol 2010;45:48-54.

18. Habashi NM. Other approaches to open-lung ventilation: airway pressure release ventilation. Crit Care Med 2005;33:S228-40.

19. Daoud EG, Farag HL, Chatburn RL. Airway pressure release ventilation: what do we know? Respir Care 2012;57:282-92.

20. Caza T, Landas S. Functional and Phenotypic Plasticity of CD4(+) T Cell Subsets. Biomed Res Int 2015;2015:521957.

21. Bajaj A, Rathor P, Kabak B. Efficacy of Airway Pressure Release Ventilation in Critically Ill Patients: A Meta-

Cite this article as: Chen C, Zhen J, Gong S, Yan J, Li L. Efficacy of airway pressure release ventilation for acute respiratory distress syndrome: a systematic review with metaanalysis. Ann Palliat Med 2021;10(10):10349-10359. doi: 10.21037/ apm-21-747
Analysis of Randomized Controlled Trials. Am J Respir Crit Care Med 2015;191:A1195.

22. Sun X, Liu Y, Li N, et al. The safety and efficacy of airway pressure release ventilation in acute respiratory distress syndrome patients: A PRISMA-compliant systematic review and meta-analysis. Medicine (Baltimore) 2020;99:e18586.

23. Carsetti A, Damiani E, Domizi R, et al. Airway pressure release ventilation during acute hypoxemic respiratory failure: a systematic review and meta-analysis of randomized controlled trials. Ann Intensive Care 2019;9:44.

24. Zhong X, Wu Q, Yang H, et al. Airway pressure release ventilation versus low tidal volume ventilation for patients with acute respiratory distress syndrome/acute lung injury: a meta-analysis of randomized clinical trials. Ann Transl Med 2020;8:1641.

25. Andrews PL, Shiber JR, Jaruga-Killeen E, et al. Early application of airway pressure release ventilation may reduce mortality in high-risk trauma patients: a systematic review of observational trauma ARDS literature. J Trauma Acute Care Surg 2013;75:635-41.

26. Jain SV, Kollisch-Singule M, Sadowitz B, et al. The 30year evolution of airway pressure release ventilation (APRV). Intensive Care Med Exp 2016;4:11.

27. Simera I, Moher D, Hoey J, et al. A catalogue of reporting guidelines for health research. Eur J Clin Invest 2010;40:35-53.

(English Language Editors: J. Goetz and J. Chapnick) 